UTTG-08-03

\title{
Can Non-Adiabatic Perturbations Arise After Single-Field Inflation?
}

\author{
Steven Weinberg ${ }^{1}$ \\ Theory Group, Department of Physics, University of Texas \\ Austin, TX, 78712
}

\begin{abstract}
It is shown that non-adiabatic cosmological perturbations cannot appear during the period of reheating following inflation with a single scalar inflaton field.
\end{abstract}

\footnotetext{
${ }^{1}$ Electronic address: weinberg@physics.utexas.edu
} 
According to a widely adopted picture [1], the perturbations to the RobertsonWalker cosmology arose from the quantum fluctuations in a slowly rolling scalar "inflaton" field during a period of inflation, then became classical as their wavelength was stretched beyond the horizon, and subsequently were imprinted on the decay products of the inflaton during a period of "reheating." One of the attractions of this picture (and in particular the assumption that there is just one inflaton field) is that it has generally been thought to lead only to adiabatic perturbations, in agreement with current observations $[2]$.

A recent preprint [3] has raised the question, whether it is possible for non-adiabatic cosmological perturbations to arise during reheating even after inflation with a single inflaton field. This would be very important if true, for then observational limits on non-adiabatic fluctuations in the cosmic microwave background would provide some constraints on the otherwise mysterious era of reheating, and indeed on the whole history of the universe between inflation and the present.

However, there are very general grounds for expecting that single-field inflation can only produce adiabatic fluctuations, whatever happens in reheating or subsequently. It has been shown [4] that, whatever the constituents of the universe, the differential equations for cosmological perturbations in Newtonian gauge always have a solution which for wavelengths outside the horizon (that is, for physical wave numbers that are much less than the cosmological expansion rate) are adiabatic, in the sense that a quantity $\zeta$ [5] is conserved:

$$
\zeta=-\Psi-\delta \rho / 3(\bar{\rho}+\bar{p})
$$

where $\delta \rho$ is the perturbation to the total energy density in Newtonian gauge; $\bar{\rho}$ and $\bar{p}$ are the unperturbed total energy density and pressure; the perturbed metric is given by

$$
d s^{2}=-(1+2 \Phi) d t^{2}+a^{2}(1-2 \Psi) d \mathbf{x}^{2}
$$

and as usual $H \equiv \dot{a} / a$. (Reference [4] dealt mostly with a quantity [6]

$$
\mathcal{R} \equiv-\Psi+H \delta u
$$

where $\delta u$ is the perturbation to the total velocity potential, but outside the horizon $\mathcal{R}$ and $\zeta$ are the same.) For this adiabatic mode, the perturbations 
to the metric and the total energy density and pressure are given outside the horizon by

$$
\begin{aligned}
& \Phi=\Psi=\zeta\left[-1+\frac{H(t)}{a(t)} \int_{t_{1}}^{t} a\left(t^{\prime}\right) d t^{\prime}\right] \\
& \delta \rho=-\frac{\zeta \dot{\bar{\rho}}(t)}{a(t)} \int_{t_{1}}^{t} a\left(t^{\prime}\right) d t^{\prime}, \quad \delta p=-\frac{\zeta \dot{\bar{p}}(t)}{a(t)} \int_{t_{1}}^{t} a\left(t^{\prime}\right) d t^{\prime} .
\end{aligned}
$$

This is a solution for any value of $t_{1}$, so the difference of adiabatic solutions with different values of $t_{1}$ is also a solution, with $\zeta=0$ and $\Phi=\Psi \propto$ $H(t) / a(t), \delta \rho / \dot{\bar{\rho}} \propto 1 / a(t)$, etc. (The freedom to choose $t_{1}$ is limited by the fact that $a(t)$ is very small early during inflation, so to avoid perturbations that are very large at early times it is necessary to take $t_{1}$ at an early time.) Furthermore, if the total energy-momentum tensor $T^{\mu \nu}$ is given by a sum of tensors $T_{\alpha}^{\mu \nu}$ for fluids labeled $\alpha$ (not necessarily individually conserved) then Eq. (5) holds for each of the individual perturbations

$$
\delta \rho_{\alpha}=-\frac{\zeta \dot{\bar{\rho}}_{\alpha}(t)}{a(t)} \int_{t_{1}}^{t} a\left(t^{\prime}\right) d t^{\prime}, \quad \delta p_{\alpha}=-\frac{\zeta \dot{\bar{p}}_{\alpha}(t)}{a(t)} \int_{t_{1}}^{t} a\left(t^{\prime}\right) d t^{\prime},
$$

and similarly for the inflaton field

$$
\delta \varphi=-\frac{\zeta \dot{\bar{\varphi}}(t)}{a(t)} \int_{t_{1}}^{t} a\left(t^{\prime}\right) d t
$$

In general there may be other solutions, for which $\zeta$ is not constant. (For no good reason these others are often called isocurvature solutions.) But if only one inflaton field makes a contribution to the energy-momentum tensor during inflation then the perturbations to this field and the metric in Newtonian gauge are governed by a second-order set of differential equations, so only two independent solutions contribute to cosmological perturbations, and since we have found two explicit solutions outside the horizon, these are the only solutions for the coupled system of inflaton and gravitational fields from horizon exit to the end of inflation. During reheating and the subsequent evolution of the universe other fields and fluids become important, but the adiabatic mode is a true solution outside the horizon whatever the contents of the universe, so with the universe in the adiabatic mode at the beginning of reheating it remains in this mode as long as the wavelength remains outside the horizon. 
So how did non-adiabatic modes arise in reference [3]? The theorem of reference [4] assumes that the field equations hold at every moment, which requires that the perturbations are differentiable functions of time. As he suggested might be the case, the reason that Armendáriz-Picón found a nonadiabatic mode during reheating in reference [3] was that, although it was assumed that initially during inflation only the inflaton field was present and there was no energy transfer to other fields, he supposed in this reference that the energy transfer rate rose discontinuously to a non-zero value at the beginning of reheating. (The model considered in reference [3] actually gave a constant value for $\zeta$, but the perturbations had unequal values of $\delta \rho / \overline{\bar{\rho}}$ for the inflaton and its decay products, so as recognized by Armendáriz-Picón, this perturbation was not adiabatic.) Of course, a discontinuous change in the energy transfer rate is unphysical.

There is one weak point in the above general argument that non-adiabatic perturbations do not arise during reheating. It is the assumption that there was nothing but the inflaton and gravitation before reheating. Of course, in order for reheating to occur at all, there must be other fields or fluids besides the inflaton, and these do not suddenly come into existence during reheating. The reactions that produce matter during reheating can't be completely absent beforehand, so the remaining question is whether the transfer of energy from the inflaton to other fields during inflation before reheating excites these other modes.

This question is answered by a further theorem, that if the matter energy density and the transfer rate of energy from inflaton to matter are both small (in a sense to be made precise below) early in inflation, then any initial nonadiabatic perturbations will decay at least as fast as $1 / a^{3}$, and will thus be exponentially small by the start of reheating.

Here is the proof. We take the co-moving rate per proper volume of energy transfer from the inflaton field $\varphi$ to "matter" fields (possibly including radiation) to be a scalar function $X$ of all these fields and perhaps their first and higher time derivatives:

$$
-u_{\mu} T_{M ; \nu}^{\mu \nu}=X(\varphi, \ldots),
$$

where $u^{\mu}$ is the four-vector velocity of the total energy-momentum tensor, normalized so that $u^{\mu} u_{\mu}=-1$. To zeroth order in all perturbations to the 
metric and other fields, this reads

$$
\dot{\bar{\rho}}_{M}+3 H\left(\bar{\rho}_{M}+\bar{p}_{M}\right)=\bar{X},
$$

where bars denote unperturbed values, taken to depend only on time. (We choose signs so that $\bar{u}^{0}=+1$.) To first order in perturbations, Eq. (8) gives in Newtonian gauge

$$
\delta \dot{\rho}_{M}+3 H\left(\delta \rho_{M}+\delta p_{M}\right)-3\left(\bar{\rho}_{M}+\bar{p}_{M}\right) \dot{\Psi}=\delta X+\Phi \bar{X},
$$

the last term on the right arising from the perturbation to $u_{0}$. We have dropped terms involving spatial gradients, which become negligible outside the horizon.

Now suppose that at some early time during inflation the density and pressure of matter and the rate of transfer of energy from the inflaton to matter are both small. (This is plausible, because the energy density of fermions and gauge fields produced by quantum fluctuations would be quadratic in the fluctuations, while the rate of energy transfer from the inflaton to matter is small as long as the inflaton is slowly rolling.) To make this assumption precise, we can introduce a small dimensionless parameter $\epsilon$, with $\bar{X}, \delta X$, $\bar{\rho}_{M}, \bar{p}_{M}, \delta \rho_{M}$, and $\delta p_{M}$ all proportional to $\epsilon$, and keep only terms of lowest order in $\epsilon$. The terms in the equations for the inflaton and gravitational field perturbations of zeroth order in $\epsilon$ then just give the equations of single-field inflation, without energy transfer to matter, and so to this order the inflaton and gravitational fields are necessarily in the adiabatic mode, described by Eqs. (4) and (7). Since $\delta X$ is already of first order in $\epsilon$, to first order it depends only on the terms in the inflaton and gravitational fields of zeroth order in $\epsilon$, and so like any other scalar quantity that depends only on these fields, the perturbation to the energy transfer rate will be given by

$$
\delta X=-\dot{\bar{X}} \mathcal{I},
$$

in which for convenience we have introduced the notation

$$
\mathcal{I}(t) \equiv \frac{\zeta}{a(t)} \int_{t_{1}}^{t} a\left(t^{\prime}\right) d t^{\prime} .
$$

(For instance, if $X$ depends only on $\varphi$, then

$$
\delta X=\frac{\partial \bar{X}}{\partial \bar{\varphi}} \delta \varphi=-\left(\frac{\partial \bar{X}}{\partial \bar{\varphi}} \dot{\bar{\varphi}}\right) \mathcal{I} .
$$


Eq. (11) also holds if $X$ depends also on $\dot{\varphi}, \ddot{\varphi}$, etc., provided that for each pair of time derivatives there is a factor of $g^{00}$ to keep $X$ a scalar.) Putting Eq. (11) together with Eq. (4), the right-hand side of Eq. (10) is

$$
\delta X+\Phi \bar{X}=-\frac{\partial}{\partial t}[\bar{X} \mathcal{I}]
$$

and Eq. (10) gives

$$
\frac{\partial}{\partial t}\left[\delta \rho_{M}+\dot{\bar{\rho}}_{M} \mathcal{I}\right]=-3 H\left(\delta \rho_{M}+\delta p_{M}\right)-3\left(\bar{\rho}_{M}+\bar{p}_{M}\right) \ddot{\mathcal{I}}-3 \frac{\partial}{\partial t}\left[\left(\bar{\rho}_{M}+\bar{p}_{M}\right) H \mathcal{I}\right] .
$$

This can be simplified by noting that $\mathcal{I}$ satisfies the differential equation

$$
\ddot{\mathcal{I}}+\frac{\partial}{\partial t}(H \mathcal{I})=0
$$

so

$$
\frac{\partial}{\partial t}\left[\delta \rho_{M}+\dot{\bar{\rho}}_{M} \mathcal{I}\right]=-3 H\left(\delta \rho_{M}+\delta p_{M}+\dot{\bar{\rho}}_{M} \mathcal{I}+\dot{\bar{p}}_{M} \mathcal{I}\right) .
$$

To continue, let us assume for the moment that the matter pressure $p_{M}$ is a function $P_{M}\left(\rho_{M}\right)$ only of the matter energy density $\rho_{M}$, as in the case of pure radiation or pure dust. (We are not assuming this for the combined system of matter and inflaton.) Then $\dot{\bar{p}}_{M}=P_{M}^{\prime}\left(\bar{\rho}_{M}\right) \dot{\bar{\rho}}_{M}$ and $\delta p_{M}=P_{M}^{\prime}\left(\bar{\rho}_{M}\right) \delta \rho_{M}$. Eq. (16) then reads

$$
\frac{\partial}{\partial t}\left[\delta \rho_{M}+\dot{\bar{\rho}}_{M} \mathcal{I}\right]=-3 H\left(1+c_{M}^{2}\right)\left(\delta \rho_{M}+\dot{\bar{\rho}}_{M} \mathcal{I}\right),
$$

where $c_{M}^{2} \equiv d P_{M} / d \rho_{M}$ is the squared sound speed. Initially $\delta \rho_{M}+\dot{\bar{\rho}}_{M} \mathcal{I}$ is small, because both $\delta \rho_{M}$ and $\dot{\bar{\rho}}_{M}$ are assumed to be small. As time passes during inflation, the transfer of energy from the inflaton to matter may make both $\delta \rho_{M}$ and $\dot{\bar{\rho}}_{M}$ large, but the particular combination $\delta \rho_{M}+\dot{\bar{\rho}}_{M} \mathcal{I}$ becomes even smaller, decaying at least as fast as $1 / a^{3}$ for $c_{M}^{2} \geq 0$, so the matter density fluctuations satisfy the adiabatic condition (6). With the matter pressure a function only of the matter density, the matter pressure perturbation will then also satisfy Eq. (6). Obviously if the energy density and pressure perturbations of matter as well as of the inflaton all satisfy Eq. (6), then the total energy density and pressure perturbations will satisfy Eq. (5). It follows that $\zeta$ will be constant, because in general

$$
\dot{\zeta}=\frac{\dot{\bar{\rho}} \delta p-\dot{\bar{p}} \delta \rho}{3(\bar{\rho}+\bar{p})^{2}}
$$


All perturbations are thus in the adiabatic mode. Eventually, the matter density perturbation will grow great enough so that it can no longer be ignored in calculating the energy transfer rate $X$ and the gravitational field perturbations, but with the density and pressure perturbations of the matter as well as the inflaton satisfying Eq. (6), we will still have $\delta X=-\dot{\bar{X}} \mathcal{I}$ and $\Phi=\Psi=-\dot{\mathcal{I}}$, so the above analysis will remain valid.

The same analysis also obviously applies if the inflaton energy goes into several species of matter, with a pressure for each species given by a function of the energy density in that species. More generally, the pressure may depend on other variables such as a particle number density $n$. The net rate of creation of particles in a co-moving frame can be assumed to be a scalar function $Y$ of the inflaton field and perhaps other fields and their time derivatives

$$
\left(n u^{\mu}\right)_{; \mu}=Y(\varphi, \ldots) \text {. }
$$

(In general there may be a difference between the particle transport velocity that should appear in this relation and the energy transport velocity $u^{\mu}$ appearing in the energy-momentum tensor, but for scalar modes the difference is a gradient, and therefore does not contribute for the long wavelengths considered here.) In the absence of perturbations, Eq. (19) gives

$$
\dot{\bar{n}}+3 H \bar{n}=\bar{Y}
$$

while to first order in perturbations, in Newtonian gauge,

$$
\delta \dot{n}+3 H \delta n-3 \bar{n} \dot{\Psi}=\delta Y+\Phi \bar{Y},
$$

where again we drop gradient terms, which are negligible outside the horizon. If $n$ as well as the matter density and pressure are small at some early time during inflation then the particle creation rate will be a scalar function only of the inflaton and gravitational perturbations and perhaps their time derivatives, which are described by the adiabatic solution (4) and (7), so just as for in Eq. (13),

$$
\delta Y+\Phi \bar{Y}=-\frac{\partial}{\partial t}[\bar{Y} \mathcal{I}]
$$

and Eq. (21) gives

$$
\frac{\partial}{\partial t}[\delta n+\dot{\bar{n}} \mathcal{I}]=-3 H[\delta n+\dot{\bar{n}} \mathcal{I}]
$$


Initially $\delta n+\dot{\bar{n}} \mathcal{I}$ is small, because both $\delta n$ and $\dot{\bar{n}}$ are assumed to be small. As time passes during inflation, the creation of particles may make both $\delta n$ and $\dot{\bar{n}}$ large, but the particular combination $\delta n+\dot{\bar{n}} \mathcal{I}$ becomes even smaller, decaying as $1 / a^{3}$, so the number density fluctuations satisfy the adiabatic condition

$$
\delta n \rightarrow-\dot{\bar{n}} \mathcal{I} \text {. }
$$

If the matter pressure is a function $P_{M}$ of the number density as well as the matter energy density, Eq. (16) will read

$\frac{\partial}{\partial t}\left[\delta \rho_{M}+\dot{\bar{\rho}}_{M} \mathcal{I}\right]=-3 H\left(1+\frac{\partial P_{M}}{\partial \rho_{M}}\right)\left(\delta \rho_{M}+\dot{\bar{\rho}}_{M} \mathcal{I}\right)-3 H\left(\frac{\partial P_{M}}{\partial n}\right)(\delta n+\dot{\bar{n}} \mathcal{I})$.

Eventually the decay of the second term on the right-hand side will leave $\delta \rho_{M}+\dot{\bar{\rho}}_{M} \mathcal{I}$ governed by Eq. (17), so it too will decay. The rest of the analysis then proceeds as before.

In conclusion, even if the decay of the inflaton to matter produces small corrections to the adiabatic solution early in inflation, these corrections will decay rather than grow as inflation proceeds, and the solution will remain adiabatic whatever other fields or particles become important during reheating.

I was greatly helped in my thinking about this problem by a correspondence some months ago with Alan Guth, and more recently by discussions with Christian Armendáriz-Picón. This research was supported by the National Science Foundation under Grant No. 0071512 and by the Robert A. Welch Foundation and also the US Navy Office of Naval Research, Grant No. N00014-03-1-0639, Quantum Optics Initiative.

\section{REFERENCES}

1. A. Guth and S.-Y Pi, Phys. Rev. Lett. 49, 1110 (1982); S. Hawking, Phys. Lett. 115B, 295 (1982); A. Starobinsky, Phys. Lett. 117B, 175 (1982); F. Bardeen, P. Steinhardt, and M. Turner, Phys. Rev. D28, 679 (1983).

2. P. Crotty, J. Garcia-Bellido, J. Lesgourgues, and A. Riazuelo, Phys. Rev. Lett. 91, 171301 (2003).

3. C. Armendáriz-Picón, astro-ph/0312389. 
4. S. Weinberg, Phys. Rev. D67, 123504 (2003). Also see the appendix to S. Weinberg, astro-ph/0306374.

5. J. M. Bardeen, P. J. Steinhardt, and M. S. Turner, Phys. Rev. D28, 679 (1983). This quantity was re-introduced by D. Wands, K. A. Malik, D. H. Lyth, and A. R. Liddle, Phys. Rev. D62, 043527 (2000). Also see D. H. Lyth, Phys. Rev. D 31, 1792 (1985); K. A. Malik, D. Wands, and C. Ungarelli, Phys. Rev. D 67, 063516 (2003).

6. J. M. Bardeen, Phys. Rev. D22, 1882 (1980); D. H. Lyth, Phys. Rev. D31, 1792 (1985). For reviews, see J. Bardeen, in Cosmology and Particle Physics, eds. Li-zhi Fang and A. Zee (Gordon \& Breach, New York, 1988); A. R. Liddle and D. H. Lyth, Cosmological Inflation and Large Scale Structure (Cambridge University Press, Cambridge, UK, 2000). 